\title{
Risk factors leading to trabeculectomy surgery of glaucoma patient using Japanese nationwide administrative claims data: a retrospective non-interventional cohort study
}

\author{
Chikako Shirai ${ }^{1}$, Satoru Tsuda ${ }^{1}$, Kunio Tarasawa ${ }^{2}$, Kiyohide Fushimi ${ }^{3}$, Kenji Fujimori $^{2^{*}}$ and Toru Nakazawa ${ }^{1 *}$ (D)
}

\begin{abstract}
Background: Early recognition and management of baseline risk factors may play an important role in reducing glaucoma surgery burdens. However, no studies have investigated them using real-world data in Japan or other countries. This study aimed to clarify the risk factors leading to trabeculectomy surgery, which is the most common procedure of glaucoma surgery, of glaucoma patient using the Japanese nationwide administrative claims data associated with the diagnosis procedure combination (DPC) system.

Methods: It was a retrospective, non-interventional cohort study. Data were collected from patients who were admitted to DPC participating hospitals, nationwide acute care hospitals and were diagnosed with glaucoma between 2012 to 2018. The primary outcome was the risk factors associated with trabeculectomy surgery. The association between baseline characteristics and trabeculectomy surgery was identified using multivariable logistic regression analysis by comparing patients with and without trabeculectomy surgery. Meanwhile, the secondary outcomes included the rate of comorbidities, the rate of concomitant drug use and the treatment patterns of glaucoma eye drops at the index admission. Among patients with trabeculectomy surgery, the risk factors leading to cataract surgery were also evaluated as subgroup analysis.

(Continued on next page)
\end{abstract}

\footnotetext{
*Correspondence: fujimori@med.tohoku.ac.jp; ntoru@oph.med.tohoku.ac.jp

${ }^{2}$ Department of Health Administration and Policy, Tohoku University

Graduate School of Medicine, 1-1 Seiryo-machi, Aoba-ku, Sendai, Miyagi 980-8574, Japan

'Department of Ophthalmology, Tohoku University Graduate School of Medicine, 1-1 Seiryo-machi, Aoba-ku, Sendai, Miyagi 980-8574, Japan

Full list of author information is available at the end of the article
}

(c) The Author(s). 2021 Open Access This article is licensed under a Creative Commons Attribution 4.0 International License, which permits use, sharing, adaptation, distribution and reproduction in any medium or format, as long as you give appropriate credit to the original author(s) and the source, provide a link to the Creative Commons licence, and indicate if changes were made. The images or other third party material in this article are included in the article's Creative Commons licence, unless indicated otherwise in a credit line to the material. If material is not included in the article's Creative Commons licence and your intended use is not permitted by statutory regulation or exceeds the permitted use, you will need to obtain permission directly from the copyright holder. To view a copy of this licence, visit http://creativecommons.org/licenses/by/4.0/. The Creative Commons Public Domain Dedication waiver (http://creativecommons.org/publicdomain/zero/1.0/) applies to the data made available in this article, unless otherwise stated in a credit line to the data. 
(Continued from previous page)

Results: A total of 29,599 patients included in the analysis, 12,038 and 17,561 patients were in the glaucoma surgery and non-glaucoma surgery cohorts, respectively. The factors associated with the increase in trabeculectomy surgery were having allergies, taking concomitant drugs including cancer, depression, ischemic heart disease and peptic ulcer, being diagnosed with primary open-angle glaucoma and longer length of stay in hospital. In contrast, the factors associated with the decrease in trabeculectomy surgery were having hypertension, taking hypertension drug, age $\geq 80$ and female.

Conclusions: Special focus on Japanese patients with glaucoma who have allergy-related comorbidities or take immune, nervous, circulatory or gastrointestinal system-related concomitant drugs seems to be desirable.

Keywords: Glaucoma, Comorbidity, Concomitant, Administrative claims data, Diagnosis procedure combination (DPC)

\section{Background}

Glaucoma is a chronic progressive optic neuropathy that can lead to irreversible blindness, affecting over 70 million adults worldwide. Intraocular pressure (IOP)-lowering therapy is the only effective strategy recognized to date [1-5].

In Japan, glaucoma is the most common cause of blindness, accounting for $28.6 \%$ of all blind regression [6]. It has an estimated prevalence of $5 \%$ in those aged over 40 years, that is, four million glaucoma patients [7]. Owing to the aging Japanese population, and glaucoma increases with age $[8,9]$, the future clinical and economic burden to the healthcare system is expected to increase.

Current therapies used to lower IOP are drug treatment (usually eye drops), laser treatment, surgical treatment, or a combination of these treatments are used [5]. Surgical treatment is considered the final step in lowering IOP due to the improvement of drug treatment, and thus, patients with glaucoma undergoing surgical treatment represent severe or drug treatment resistance. Nevertheless, glaucoma surgery has incrementally advanced over the years. The search for safer and less invasive surgeries has been continued with emphasis on newer devices and techniques that use small incisions, a category described as micro-invasive glaucoma surgery [10-12]. New procedures for surgical treatment, however, have their own risks and complications, some of which might be unknown until long-term data become available.

Besides, to the best of our knowledge, there have been no published study results analyzing what background of patients with glaucoma who are receiving surgical treatment in routine clinical practice. Therefore, such study, in particular an analysis of the glaucoma treatment using a large-scale administrative database, resulted in an important and useful information that reflects the current status of treatment and identifies issues to be considered.
The aim of this study was to clarify the risk factors leading to trabeculectomy surgery of glaucoma patients using the Japanese nationwide administrative claims data associated with the diagnosis procedure combination (DPC) system [13, 14]. In addition, the rate of comorbidities, the rate of concomitant drug use, and the treatment patterns of glaucoma eye drops at the index admission in patients with and without trabeculectomy surgery were also explored.

\section{Methods \\ Study design}

This was a retrospective non-interventional cohort study using 6-year data (between 1 April 2012 and 31 March 2018) from the Japanese nationwide administrative claims data associated with the DPC system to identify the risk factors leading to trabeculectomy surgery of patients with glaucoma in Japan. Moreover, because glaucoma and cataract are leading causes of blindness worldwide and their co-existence is common in elderly people, the subgroup analysis was performed in patients with combined trabeculectomy and cataract surgery.

This study was registered with UMIN Clinical Trials (UMIN000037878). It was approved by the research ethics committee of the Tohoku University Graduate School of Medicine, Japan (No. 2019-1-897) and conducted in accordance with The Code of Ethics of the World Medical Association (Declaration of Helsinki) for experiments involving human. Because this study was based on secondary analysis of DPC data that had already been anonymized unlinkable, written informed consent from patients was not required.

\section{Data source and study cohort}

The DPC is a national administrative database of a casemix classification system for acute inpatient care developed in Japan. Details of the system have been described elsewhere [15, 16]. The system was launched in 2003 among 82 special functional hospitals, with a rapidly 
increasing number of hospitals having adopted the system, which recently includes approximately 7 million inpatients per year from more than 1000 hospitals, covering approximately $90 \%$ of all hospitalization to acute care hospital in Japan. The DPC data include administrative claims data and some clinical data. The DPC database includes data on the following elements: patient demographics (e.g., age and gender); primary diagnosis; comorbidities at admission; complications after admission; procedures including surgery, medication and devices used during hospitalization; length of stay; discharge status; and medical expenses [17-19]. The primary diagnosis is limited to one. In order to optimize the accuracy of the recorded diagnoses, the responsible physicians are required to record the diagnosed disease name in the medical charts. A wide variety of centers, including academic, urban and rural hospitals, use the DPC system [20]. These data were coded in the 10th revision of the International Statistical Classification of Disease (ICD10) and also the original Japanese code determined by the Ministry of Health, Labor and Welfare of Japan.

For this study, patients were extracted from the DPC data using SQL Server 2014 Management Studio (Microsoft Corporation). Eligible patients were those who were admitted to DPC participating hospitals, that is, nationwide acute care hospitals in Japan and diagnosed with glaucoma. Patients with glaucoma as the disease associated with the code of "highest medical costs" in the DPC database, it means that the most causative disease of hospitalization, were identified based on the following ICD10codes: H401 (normal tension glaucoma, primary open-angle glaucoma and open-angle glaucoma) and H409 (unspecified glaucoma) from 1 April 2012 to 31 March 2018 (study period). The less common types including angle closer glaucoma (H402), secondary glaucoma (H403-H407) and other glaucoma (H408) were excluded. Figure 1 shows the patient selection process. Trabeculectomy surgery was defined as the following claim code for medical procedure: 150335910 (trabeculectomy, TLE). The trabeculotomy (TLO), as well as MIGS and implants, is often additionally used during cataract surgery, and is unlikely to be an indicator of severe glaucoma, while TLE is a standard surgery for glaucoma in the world. More effective in lowering IOP than TLO and rarely used for an additional surgery. To obtain an appropriate outcome of this study, we selected only TLE among glaucoma surgery (GS). The patients with glaucoma who had $\geq 2$ records of GS, readmission to the DPC hospitals, < 12 months post-index continuous enrollment, in-hospital death or missing data during the study period were excluded, and furthermore, this study used the strict inclusion criteria that excluded data for the first and last year during the study period. The patients with only 1 record of TLE from 1 April 2013 to 31 March 2017 was defined as the newly undergone TLE (patients with TLE [GS cohort]).

On the other hand, patients who had not undergone TLE and all other GS (patients without GS [non-GS cohort]) were defined as those who had no record of GS during the study period and who had no record of GS from 1 April 2013 to 31 March 2017. Furthermore, to maintain the independence of observation and to exclude readmission to the DPC hospital, only the data associated with the last admission (index admission) from 1 April 2013 to 31 March 2017 were used as the data of the patients without GS.

Among the patients with TLE, those who have undergone cataract surgery (CS) were defined as patients with CS (CS cohort). CS was defined as the following claim code for medical procedure: 150253010, 190179210, 190195910 (all 3 codes related to lens reconstruction). On the other hand, persons who had not undergone CS were defined as patients without CS (non-CS cohort).

\section{Variables}

Variable used in this study included age, gender, body mass index (BMI), smoking index, season at admission, length of stay in hospital (LOS), Charlson comorbidity index (CCI) [21, 22], comorbidities at index admission (17 disease as the possible risk factors for glaucoma: [23-26] hypertension, hypotension, ischemic heart disease, heart failure, stroke, diabetes, hyperlipidemia, electrolyte disorders, thyroid dysfunction, systemic lupus erythematosus, dementia, depression, mental disorders, cancer, allergies, peptic ulcer and liver insufficiency/failure), concomitant drugs at index admission (systemic oral drugs for the 17 disease listed above) and glaucoma related measure including glaucoma types, topical glaucoma drugs at index admission (see Table 1 for medical and pharmacy codes). Comorbidities and concomitant drugs were identified based on the medical code and the pharmacy code, respectively. Concomitant drugs used in the day of surgery were excluded. Topical glaucoma treatments included prostaglandin analog (PG), $\beta$ blocker (BB), carbonic anhydrase inhibitor (CAI), $\alpha 2$ agonist (AA), $\alpha 1$ blocker (AB), $\alpha \beta$ blocker (ABB), sympathomimetics, rho-associated coiled kinase inhibitor (ROCKI), fixed-combinations and their generics. Patients were categorized into 6 age groups: $\leq 39,40-49,50-59$, 60-69, 70-79, $\geq 80$; two gender groups: male, female; four BMI groups: thin $(<18.5)$, normal $(\geq 18.5<25)$, fat $(\geq 25)$, not classified; four season groups: spring (AprilJune), summer (July-September), autumn (October-December), winter (January-March); 4 CCI groups: low (0), medium $(1,2)$, high $(3,4)$, very high $(\geq 5)$ cases. 


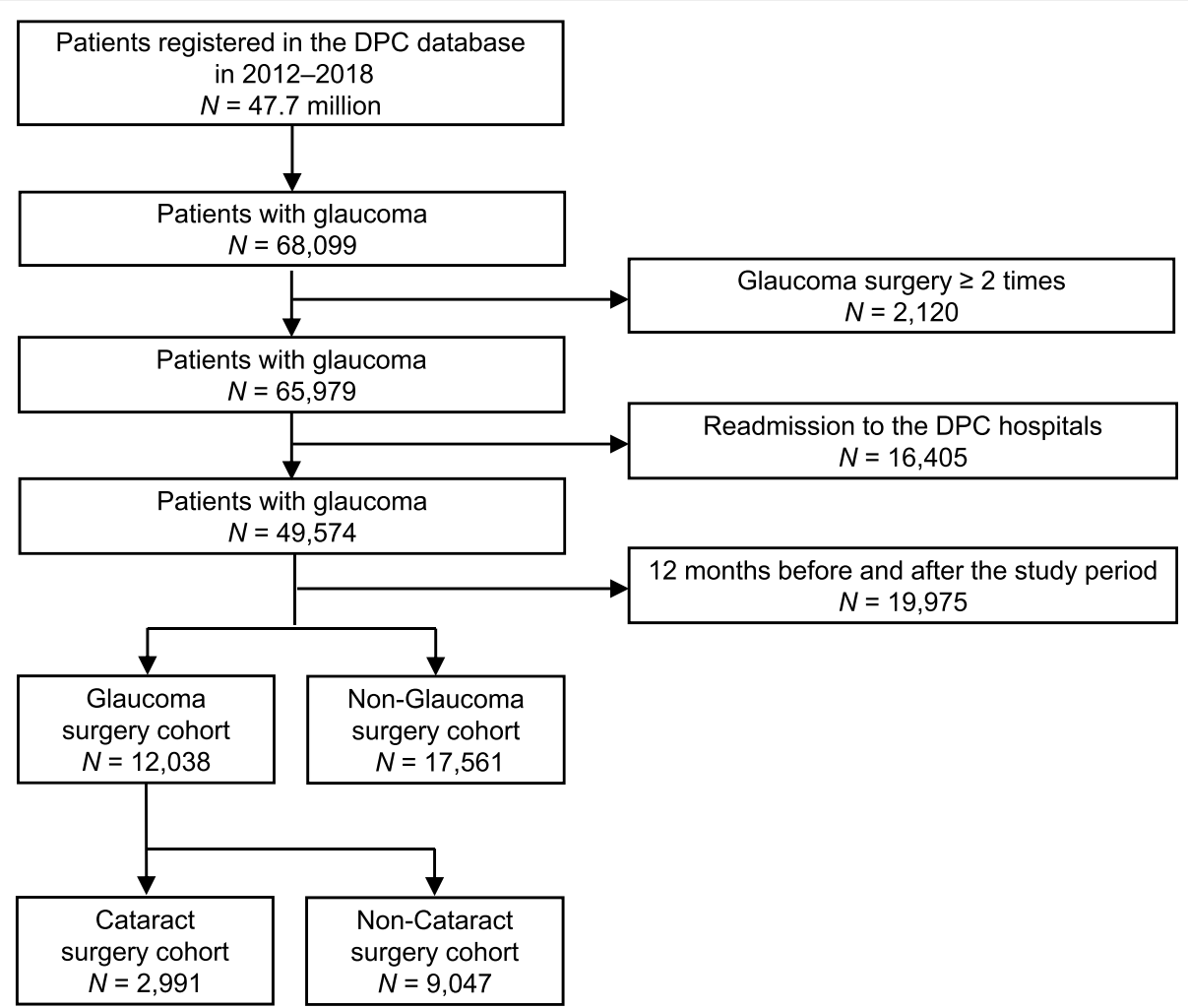

Fig. 1 Flowchart for data extraction

\section{Study outcomes}

The primary outcome was the risk factors associated with TLE using patients' baseline demographic and clinical characteristics at the index admission. The secondary outcomes were the rate of comorbidities, the rate of concomitant use of prescribed systemic oral drugs and the treatment patterns of prescribed topical glaucoma drugs (eye drops) at the index admission in the GS and non-GS cohorts. Furthermore, GS cohort was divided into two cohorts based on with or without CS. The primary and secondary outcomes were also performed.

\section{Statistical analysis}

Descriptive analysis was performed for the basic features of the data as in mean with standard deviation (SD) for continuous variables and frequency (n) and percentage (\%) for categorical variables. For outcomes, the difference between the GS and non-GS cohorts were compared according to baseline characteristics. Based on variable types and normality, the Chi-square test (or Fisher's exact test, if cell expectations were less than 5), the Student's t-test and the Mann-Whitney U test were used to examine differences.

To identify the factors associated with TLE, logistic regression analysis was used. Potentially significant variables on univariable analysis $(p<0.05)$ were considered a priori for inclusion in a multivariable logistic regression model. Pairwise correlation coefficients were examined between variables that were potentially related before inclusion in our multivariable model to avoid collinearity. Stepwise forward-backward elimination analysis was performed and variables with $p<0.0001$ were retained in the final multivariable model. In order to validate the final model, the variance inflation factor (VIF) was performed to test for multicollinearity among the predictor variables. A VIF exceeding 10 was regarded as indicating serious multicollinearity, and values greater than 4.0 was considered a cause for concern [27-29]. $P$ -values, adjusted odds ratios (ORs) and Wald 95\% confidence intervals (CIs) were obtained for the predictor variables. Furthermore, stratified analysis by CS were also performed. All the statistical analyses were performed using the JMP Pro Ver 14.0 (SAS Institute Inc., Cary, NC, USA). $P$-values of $<0.05$ were considered statistically significant.

\section{Results}

A total of 29,599 patients met all inclusion criteria for this study, of whom 12,038 (40.7\%) patients were in the GS cohort and 17,561 (59.3\%) patients were in the non- 
Table 1 Medical and pharmacy codes used for the study

\begin{tabular}{|c|c|c|c|}
\hline $\begin{array}{l}\text { Therapeutic } \\
\text { category }\end{array}$ & ICD-10 codes & Drug codes $^{a}$ & Drug generic name \\
\hline Hypertension & 110 & $213,214,217$ & \\
\hline Hypotension & $195 \%$ & 216 & \\
\hline $\begin{array}{l}\text { Ischemic heart } \\
\text { disease }\end{array}$ & $120 \%-125 \%$ & $212,214,217$ & \\
\hline Heart failure & $150 \%$ & $211,213,214,217$ & \\
\hline Stroke & $163 \%$ & 333,339 & \\
\hline Diabetes & E10\%-E14\% & 396 & \\
\hline Hyperlipidemia & E78\% & 218 & \\
\hline Electrolyte disorders & E87\% & 321,322 & \\
\hline Thyroid dysfunction & E02\%, E03\% & 243 & \\
\hline $\begin{array}{l}\text { Systemic lupus } \\
\text { erythematosus }\end{array}$ & M32\% & $\begin{array}{l}2,456,001,3,999,005 \\
3,999,038\end{array}$ & $\begin{array}{l}\text { Prednisolone, Azathioprine, } \\
\text { Hydroxychloroquine }\end{array}$ \\
\hline Depression & F251, F31\%-F35\% & 1179 & \\
\hline Dementia & F00\%-F03\% & $\begin{array}{l}1,190,012,1,190,018 \\
1,190,019\end{array}$ & $\begin{array}{l}\text { Donepezil, Memantine, } \\
\text { Galantamine, }\end{array}$ \\
\hline Mental disorders & F09, F23, F28, F29 & 117 & \\
\hline Cancer & C00\%-С97\% & $\begin{array}{l}421,422,423,424 \\
429\end{array}$ & \\
\hline Allergies & $\begin{array}{l}\text { D690, D721, H011, H101, H169, H200, H650, H651, H654, H659, J301- } \\
\text { J304, L23\%, L500, T781, T784 }\end{array}$ & $441,442,443,449$ & \\
\hline Peptic ulcer & F54, K221, K25\%, K26\%, K27\%, K28\%, K51\%, K626, K633, K828, K838 & 232 & \\
\hline $\begin{array}{l}\text { Liver insufficiency/ } \\
\text { failure }\end{array}$ & $\mathrm{K} 70 \%-\mathrm{K} 77 \%$ & $\begin{array}{l}625,6399,3919 \\
3999\end{array}$ & \\
\hline $\begin{array}{l}\text { Glaucoma (NTG/ } \\
\text { POAG/OAG) }\end{array}$ & H401 & $131^{\mathrm{b}}$ & \\
\hline $\begin{array}{l}\text { Unspecified } \\
\text { glaucoma }\end{array}$ & $\mathrm{H} 409$ & $131^{\mathrm{b}}$ & \\
\hline
\end{tabular}

ICD-10 international classification of disease, NTG normal tension glaucoma, OAG open angle glaucoma, POAG primary open angle glaucoma

${ }^{a}$ Classified by the Drug Therapeutic Class Code in Japan

${ }^{b}$ Furthermore, commonly prescribed glaucoma eye drops were narrowed down by the Japanese National Health Insurance drug price listing pharmaceuticals code

GS cohorts (Fig. 1). The non-GS cohort was considered to be patients who were hospitalized because of the sudden increase in IOP, however, cured and discharged only by drug treatment. Furthermore, it might have been hospitalized for a glaucoma medical checkup. Among the GS cohort, 2991 (24.8\%) patients were in the CS cohort and $9047(75.2 \%)$ patients were in the non-CS cohorts.

The baseline demographics and clinical characteristics of the GS and non-GS cohorts are summarized in Table 2. The mean age was 68 years and $55.6 \%$ were male, and most patients had a moderate BMI and a low CCI. In unadjusted comparison, the GS cohort significantly had a higher percentage of patients with age 5059 or $60-69(P<0.0001$ each); male $(P<0.0001)$; normal BMI $(P=0.0313)$; longer LOS $(P<0.0001)$; 2 comorbidities at the index admission: diabetes $(P=0.0101)$ and allergies $(P<0.0001) ; 11$ concomitant drugs at index admission: ischemic heart disease, stroke, diabetes, systemic lupus erythematosus, depression, mental disorders, cancer, allergies, peptic ulcer $(P<0.0001$ each), hyperlipidemia $(P=0,0016)$ and liver insufficiency/failure $(P=$ $0.0294)$; and a diagnosis of primary open angle glaucoma (POAG) $(P<0.0001)$ than the non-GS cohort, whereas significantly had a lower percentage of patients with age $\leq 39$ or $\geq 80 \quad(P<0.0001$ each $)$; female $(P<0.0001)$; autumn season admission $(P=0.0041) ; 1$ comorbidity at index admission: hypertension $(P=0.0009)$ : 2 concomitant drugs at index admission: hypertension and heart failure $(P<0.0001$ each); and a diagnosis of normal tension glaucoma (NTG) $(P<0.0001)$. Concerning the season of hospital admission, we thought that IOP rises in winter and trabeculectomy would be more frequent, however, medical big data such as the Japanese nationwide database which has more than forty million acute care hospitalized patients registered, did not show seasonal difference.

Regarding the primary outcome on the risk factors associated with TLE, the 28 variables previously 
Table 2 Baseline demographic and clinical characteristics of the study cohorts

\begin{tabular}{|c|c|c|c|c|c|}
\hline \multirow[t]{2}{*}{ Variable } & \multicolumn{2}{|c|}{$\begin{array}{l}\text { Glaucoma surgery } \\
(N=12,038)\end{array}$} & \multicolumn{2}{|c|}{$\begin{array}{l}\text { Non-glaucoma surgery } \\
(N=17,561)\end{array}$} & \multirow[b]{2}{*}{$P$ Value } \\
\hline & $n$ & $\begin{array}{l}\text { Percentage or } \\
\text { mean } \pm S D\end{array}$ & $n$ & $\begin{array}{l}\text { Percentage or } \\
\text { mean } \pm S D\end{array}$ & \\
\hline Age (years) & 12,038 & $68.5 \pm 12.4$ & 17,561 & $68.8 \pm 14.0$ & $<0.0001^{*}$ \\
\hline Category & & & & & $<0.0001$ \\
\hline$\leq 39$ & 305 & 2.5 & 719 & 4.1 & $<0.0001$ \\
\hline $40-49$ & 628 & 5.2 & 929 & 5.3 & 0.7910 \\
\hline $50-59$ & 1488 & 12.4 & 1842 & 10.5 & $<0.0001$ \\
\hline $60-69$ & 3227 & 26.8 & 4168 & 23.7 & $<0.0001$ \\
\hline $70-79$ & 4207 & 34.9 & 6069 & 64.6 & 0.4943 \\
\hline$\geq 80$ & 2183 & 18.1 & 3034 & 21.8 & $<0.0001$ \\
\hline \multicolumn{6}{|l|}{ Gender } \\
\hline Category & & & & & $<0.0001$ \\
\hline Male & 7032 & 58.4 & 9376 & 53.4 & $<0.0001$ \\
\hline Female & 5006 & 41.6 & 8185 & 46.6 & $<0.0001$ \\
\hline Body mass index $\left(\mathrm{kg} / \mathrm{m}^{2}\right)$ & 12,038 & $22.7 \pm 4.3$ & 17,561 & $22.2 \pm 6.2$ & $<0.0001^{*}$ \\
\hline Category & & & & & $<0.0001$ \\
\hline Thin $(<18.5)$ & 1076 & 8.9 & 1565 & 8.9 & 0.9504 \\
\hline Normal $(\geq 18.5<25)$ & 7867 & 65.4 & 11,262 & 64.1 & 0.0313 \\
\hline Fat $(\geq 25)$ & 2969 & 24.7 & 4220 & 24.0 & 0.2144 \\
\hline Not classified & 126 & 1.0 & 514 & 2.9 & $<0.0001$ \\
\hline Smoking index & 12,038 & $91.3 \pm 288.5$ & 17,561 & $89.9 \pm 290.614 .0$ & $0.9622^{*}$ \\
\hline Maximum & 0 & & 0 & & \\
\hline Minimum & 5000 & & 4995 & & \\
\hline \multicolumn{6}{|l|}{ Season } \\
\hline Category & & & & & 0.0303 \\
\hline Spring (April-June) & 3295 & 27.4 & 4659 & 26.5 & 0.1093 \\
\hline Summer (July-September) & 2504 & 20.8 & 3644 & 20.8 & 0.9187 \\
\hline Autumn (October-December) & 2520 & 20.9 & 3922 & 22.3 & 0.0041 \\
\hline Winter (January-March) & 3719 & 30.9 & 5336 & 30.4 & 0.3553 \\
\hline Length of stay in hospital & 12,038 & $12.1 \pm 6.1$ & 17,561 & $8.6 \pm 6.0$ & $<0.0001^{*}$ \\
\hline Maximum & 1 & & 1 & & \\
\hline Minimum & 99 & & 128 & & \\
\hline \multicolumn{6}{|l|}{ Charlson comorbidity index } \\
\hline Category & & & & & 0.4710 \\
\hline Low (0) & 9748 & 81.0 & 14,287 & 81.4 & 0.4135 \\
\hline Medium $(1,2)$ & 2177 & 18.1 & 3100 & 17.7 & 0.3457 \\
\hline High $(3,4)$ & 109 & 0.9 & 162 & 0.9 & 0.9013 \\
\hline Very High ( $\geq 5$ ) & 4 & 0.0 & 12 & 0.1 & 0.3085 \\
\hline \multicolumn{6}{|l|}{ Comorbidities } \\
\hline \multicolumn{6}{|l|}{ Circulatory system } \\
\hline Hypertension & 852 & 7.1 & 1428 & 8.1 & 0.0009 \\
\hline Hypotension & 2 & 0.0 & 5 & 0.0 & 0.7820 \\
\hline Ischemic heart disease & 292 & 2.4 & 412 & 2.3 & 0.6694 \\
\hline Heart failure & 82 & 0.7 & 147 & 0.8 & 0.1377 \\
\hline
\end{tabular}


Table 2 Baseline demographic and clinical characteristics of the study cohorts (Continued)

\begin{tabular}{|c|c|c|c|c|c|}
\hline \multirow[t]{2}{*}{ Variable } & \multicolumn{2}{|c|}{$\begin{array}{l}\text { Glaucoma surgery } \\
(N=12,038)\end{array}$} & \multicolumn{2}{|c|}{$\begin{array}{l}\text { Non-glaucoma surgery } \\
(N=17,561)\end{array}$} & \multirow[b]{2}{*}{$P$ Value } \\
\hline & $n$ & $\begin{array}{l}\text { Percentage or } \\
\text { mean } \pm S D\end{array}$ & $n$ & $\begin{array}{l}\text { Percentage or } \\
\text { mean } \pm S D\end{array}$ & \\
\hline Stroke & 80 & 0.7 & 118 & 0.7 & 1.0000 \\
\hline \multicolumn{6}{|l|}{ Metabolic system } \\
\hline Diabetes & 1763 & 14.6 & 2386 & 13.6 & 0.0101 \\
\hline Hyperlipidemia & 385 & 3.2 & 575 & 3.3 & 0.7166 \\
\hline Electrolyte disorders & 134 & 1.1 & 232 & 1.3 & 0.1204 \\
\hline Thyroid dysfunction & 14 & 0.1 & 28 & 0.2 & 0.3512 \\
\hline Systemic lupus erythematosus & 10 & 0.1 & 28 & 0.2 & 0.0971 \\
\hline \multicolumn{6}{|l|}{ Nervous system } \\
\hline Dementia & 18 & 0.2 & 43 & 0.2 & 0.0894 \\
\hline Depression & 56 & 0.5 & 88 & 0.5 & 0.7339 \\
\hline Mental disorders & 1 & 0.0 & 0 & 0.0 & 0.4067 \\
\hline \multicolumn{6}{|l|}{ Immune system } \\
\hline Cancer & 110 & 0.9 & 155 & 0.9 & 0.8017 \\
\hline Allergies & 981 & 8.1 & 542 & 3.1 & $<0.0001$ \\
\hline \multicolumn{6}{|l|}{ Gastrointestinal system } \\
\hline Peptic ulcer & 181 & 1.5 & 221 & 1.3 & 0.0821 \\
\hline Liver insufficiency/failure & 30 & 0.2 & 35 & 0.2 & 0.3784 \\
\hline \multicolumn{6}{|l|}{ Concomitant drug } \\
\hline \multicolumn{6}{|l|}{ Circulatory system } \\
\hline Hypertension & 3416 & 28.4 & 5546 & 31.6 & $<0.0001$ \\
\hline Hypotension & 36 & 0.3 & 56 & 0.3 & 0.8319 \\
\hline Ischemic heart disease & 2125 & 17.7 & 2625 & 14.9 & $<0.0001$ \\
\hline Heart failure & 3656 & 30.4 & 5735 & 32.7 & $<0.0001$ \\
\hline Stroke & 2942 & 24.4 & 3053 & 17.4 & $<0.0001$ \\
\hline \multicolumn{6}{|l|}{ Metabolic system } \\
\hline Diabetes & 588 & 4.9 & 635 & 3.6 & $<0.0001$ \\
\hline Hyperlipidemia & 777 & 6.5 & 977 & 5.6 & 0.0016 \\
\hline Electrolyte disorders & 1233 & 10.2 & 1907 & 10.9 & 0.0909 \\
\hline Thyroid dysfunction & 78 & 0.6 & 106 & 0.6 & 0.6518 \\
\hline Systemic lupus erythematosus & 303 & 2.5 & 301 & 1.7 & $<0.0001$ \\
\hline \multicolumn{6}{|l|}{ Nervous system } \\
\hline Dementia & 45 & 0.4 & 84 & 0.5 & 0.2084 \\
\hline Depression & 2518 & 20.9 & 2005 & 11.4 & $<0.0001$ \\
\hline Mental disorders & 2522 & 21.0 & 2012 & 11.5 & $<0.0001$ \\
\hline \multicolumn{6}{|l|}{ Immune system } \\
\hline Cancer & 9313 & 77.4 & 3997 & 22.8 & $<0.0001$ \\
\hline Allergies & 436 & 3.6 & 433 & 2.5 & $<0.0001$ \\
\hline \multicolumn{6}{|l|}{ Gastrointestinal system } \\
\hline Peptic ulcer & 336 & 18.5 & 2450 & 14.9 & $<0.0001$ \\
\hline Liver insufficiency/failure & 2230 & 2.8 & 418 & 2.4 & 0.0294 \\
\hline \multicolumn{6}{|l|}{ Glaucoma types } \\
\hline POAG & 4404 & 36.6 & 5628 & 32.0 & $<0.0001$ \\
\hline
\end{tabular}


Table 2 Baseline demographic and clinical characteristics of the study cohorts (Continued)

\begin{tabular}{|c|c|c|c|c|c|}
\hline \multirow[t]{2}{*}{ Variable } & \multicolumn{2}{|c|}{$\begin{array}{l}\text { Glaucoma surgery } \\
(N=12,038)\end{array}$} & \multicolumn{2}{|c|}{$\begin{array}{l}\text { Non-glaucoma surgery } \\
(N=17,561)\end{array}$} & \multirow[b]{2}{*}{$P$ Value } \\
\hline & $n$ & $\begin{array}{l}\text { Percentage or } \\
\text { mean } \pm S D\end{array}$ & $n$ & $\begin{array}{l}\text { Percentage or } \\
\text { mean } \pm S D\end{array}$ & \\
\hline OAG & 4214 & 35.0 & 6300 & 36.0 & 0.1253 \\
\hline NTG & 743 & 6.2 & 1331 & 7.6 & $<0.0001$ \\
\hline Not classified & 2677 & 22.2 & 4320 & 24.5 & $<0.0001$ \\
\hline \multicolumn{6}{|l|}{ Glaucoma drug by class } \\
\hline PG & 2827 & 23.5 & 3923 & 22.3 & 0.0215 \\
\hline BB & 713 & 5.9 & 1212 & 6.9 & 0.0008 \\
\hline CAl & 1086 & 9.0 & 1445 & 8.2 & 0.0168 \\
\hline ROCKI & 694 & 5.8 & 928 & 5.3 & 0.0771 \\
\hline AA & 1640 & 13.3 & 2317 & 13.2 & 0.7534 \\
\hline$A B$ & 131 & 1.1 & 141 & 0.8 & 0.0130 \\
\hline$A B B$ & 14 & 0.1 & 17 & 0.1 & 0.7151 \\
\hline Sympathomimetics & 12 & 0.1 & 11 & 0.1 & 0.2655 \\
\hline PG/BB fixed combination & 296 & 2.5 & 425 & 2.4 & 0.8479 \\
\hline CAI/BB fixed combination & 713 & 5.9 & 1212 & 6.9 & 0.0008 \\
\hline \multicolumn{6}{|l|}{ Glaucoma drug by generic name } \\
\hline \multicolumn{6}{|l|}{ PG } \\
\hline Isopropyl Unoprostone & 4 & 0.0 & 13 & 0.1 & 0.2165 \\
\hline Isopropyl Unoprostone GE & 0 & 0.0 & 0 & 0.0 & NA \\
\hline Latanoprost & 917 & 7.6 & 1272 & 7.2 & 0.2309 \\
\hline Latanoprost GE & 152 & 1.3 & 238 & 1.4 & 0.5050 \\
\hline Travoprost & 377 & 3.1 & 550 & 3.1 & 1.0000 \\
\hline Travoprost GE & 0 & 0.0 & 0 & 0.0 & NA \\
\hline Tafluprost & 367 & 3.0 & 669 & 3.8 & 0.0004 \\
\hline Tafluprost GE & 0 & 0.0 & 0 & 0.0 & NA \\
\hline Bimatoprost & 1069 & 8.9 & 1274 & 7.3 & $<0.0001$ \\
\hline Bimatoprost GE & 0 & 0.0 & 0 & 0.0 & NA \\
\hline \multicolumn{6}{|l|}{ BB } \\
\hline Timolol Maleate & 435 & 3.6 & 761 & 4.3 & 0.0020 \\
\hline Timolol Maleate GE & 52 & 0.4 & 73 & 0.4 & 0,8544 \\
\hline Carteolol Hydrochloride & 217 & 1.8 & 367 & 2.1 & 0.0813 \\
\hline Carteolol Hydrochloride GE & 10 & 0.1 & 12 & 0.1 & 0.6687 \\
\hline Betaxolol Hydrochloride & 2 & 0.0 & 5 & 0.0 & 0.7082 \\
\hline Betaxolol Hydrochloride GE & 0 & 0.0 & 1 & 0.0 & 1.0000 \\
\hline \multicolumn{6}{|l|}{$\mathrm{CAl}$} \\
\hline Dorzolamide Hydrochloride & 225 & 1.9 & 295 & 1.7 & 0.2243 \\
\hline Dorzolamide Hydrochloride GE & 0 & 0.0 & 0 & 0.0 & NA \\
\hline Brinzolamide & 686 & 5.7 & 914 & 5.2 & 0.0670 \\
\hline Brinzolamide GE & 0 & 0.0 & 0 & 0.0 & NA \\
\hline \multicolumn{6}{|l|}{ ROCKI } \\
\hline Ripasudil Hydrochloride Hydrate & 694 & 5.8 & 928 & 5.3 & 0.0771 \\
\hline Ripasudil Hydrochloride Hydrate GE & 0 & 0.0 & 0 & 0.0 & NA \\
\hline
\end{tabular}


Table 2 Baseline demographic and clinical characteristics of the study cohorts (Continued)

\begin{tabular}{|c|c|c|c|c|c|}
\hline \multirow[t]{2}{*}{ Variable } & \multicolumn{2}{|c|}{$\begin{array}{l}\text { Glaucoma surgery } \\
(N=12,038)\end{array}$} & \multicolumn{2}{|c|}{$\begin{array}{l}\text { Non-glaucoma surgery } \\
(N=17,561)\end{array}$} & \multirow[b]{2}{*}{$P$ Value } \\
\hline & $\bar{n}$ & $\begin{array}{l}\text { Percentage or } \\
\text { mean } \pm S D\end{array}$ & $\bar{n}$ & $\begin{array}{l}\text { Percentage or } \\
\text { mean } \pm S D\end{array}$ & \\
\hline Brimonidine Tartrate & 1604 & 13.3 & 2317 & 13.2 & 0.7534 \\
\hline Brimonidine Tartrate GE & 0 & 0.0 & 0 & 0.0 & NA \\
\hline \multicolumn{6}{|l|}{$A B$} \\
\hline Bunazosin Hydrochloride & 131 & 1.1 & 141 & 0.8 & 0.0130 \\
\hline Bunazosin Hydrochloride GE & 0 & 0.0 & 0 & 0.0 & NA \\
\hline \multicolumn{6}{|l|}{$A B B$} \\
\hline Levobunolol Hydrochloride & 2 & 0.0 & 1 & 0.0 & 0.5705 \\
\hline Levobunolol Hydrochloride GE & 0 & 0.0 & 0 & 0.0 & NA \\
\hline Nipradilol & 13 & 0.1 & 16 & 0.1 & 0.7067 \\
\hline Nipradilol GE & 0 & 0.0 & 1 & 0.0 & 1.0000 \\
\hline \multicolumn{6}{|l|}{ Sympathomimetics } \\
\hline Dipivefrin Hydrochloride & 12 & 0.1 & 11 & 0.1 & 0.2917 \\
\hline Dipivefrin Hydrochloride GE & 0 & 0.0 & 0 & 0.0 & NA \\
\hline \multicolumn{6}{|l|}{ PG/BB fixed combination } \\
\hline Lat/Tim & 114 & 0.9 & 211 & 1.2 & 0.0409 \\
\hline Lat/Tim GE & 0 & 0.0 & 0 & 0.0 & NA \\
\hline Lat/Car & 1 & 0.0 & 3 & 0.0 & 0.6560 \\
\hline Lat/Car GE & 0 & 0.0 & 0 & 0.0 & NA \\
\hline Tra/Tim & 141 & 1.2 & 163 & 0.9 & 0.0459 \\
\hline Tra/Tim GE & 0 & 0.0 & 0 & 0.0 & NA \\
\hline Taf/Tim & 41 & 0.3 & 51 & 0.3 & 0.4582 \\
\hline Taf/Tim GE & 0 & 0.0 & 0 & 0.0 & NA \\
\hline \multicolumn{6}{|l|}{ CAI/BB fixed combination } \\
\hline Dor/Tim & 1519 & 12.6 & 2327 & 13.3 & 0.1133 \\
\hline Dor/Tim GE & 0 & 0.0 & 0 & 0.0 & NA \\
\hline Brinzolamide/Tim & 359 & 3.0 & 547 & 3.1 & 0.5364 \\
\hline Brinzolamide/Tim GE & 0 & 0.0 & 0 & 0.0 & NA \\
\hline
\end{tabular}

$A A$ a2-agonist, $A B$ a1-blocker, $A B B$ aß-blocker, $B B$ B-blocker, $C A l$ carbonic anhydrase inhibitor, Dor dorzolamide hydrochloride, $G E$ generic, $L a t$ latanoprost, $N A$ not assessed, NTG normal tension glaucoma, OAG open angle glaucoma, PG prostaglandin analog, POAG primary open angle glaucoma, ROCKI rho-associated protein kinase inhibitor, SD standard deviation, Taf tafluprost, Tim timolol maleate, Tra travoprost

${ }^{*}$ Calculated using the Mann-Whitney $\mathrm{U}$ test; the remaining $P$ Values were calculated with the Chi-square test or Fisher's exact test

mentioned were included in the initial regression model. Stepwise logistic regression with forward-backward elimination retained 11 of the 20 variables as the significant predictors $(P<0.0001)$. The VIFs for the predictor variables in this study were all $<4.0$, indicating the absence of multicollinearity. Table 3 lists the variables estimated for the final model. The use of cancer drug (adjusted OR: 0.0862, 95\% CI: 0.0816-0.0911) and having allergies including systemic and topical (adjusted OR: 0.3590, 95\% CI: 0.3223-0.3997) were the most significant predictors of TLE, followed by using concomitant drugs including depression, ischemic heart disease and peptic ulcer; being diagnosed with POAG; and longer LOS. In contrast, the use of hypertension drug (adjusted OR: 1.1651, 95\% CI: 1.1073-1.2258) and having hypertension (adjusted OR: 1.1621, 95\% CI: 1.0640-1.2693) was most strongly associated with reduced likelihood of TLE followed by age $\geq 80$ and female. When we restricted the multivariable analysis to the subgroup of patients with TLE who had undergone CS, the association between increase CS and baseline characteristics $(P<0.0001)$; age $70-79$ and being diagnosed with POAG were also significant in the model (Table 4).

The rate of comorbidities and the rate of concomitant use of prescribed systemic oral drugs in the GS and non-GS cohorts are shown in Fig. 2. The most common 
Table $\mathbf{3}$ Logistic regression analysis of factors associated with or without trabeculectomy surgery

\begin{tabular}{llll}
\hline Variable & Estimate & Adjusted OR (95\% Cl) & $P$ Value \\
\hline Hypertension drug & 0.649155 & $1.1651(1.1073-1.2258)$ & $<0.0001$ \\
Hypertension & 0.33362 & $1.1621(1.0640-1.2693)$ & $<0.0001$ \\
Age $\geq 80$ & 0.174507 & $1.2609(1.0189-1.3370)$ & $<0.0001$ \\
Female & 0.151442 & $1.2263(1.1702-1.2851)$ & $<0.0001$ \\
Length of stay in hospital & -0.06979 & $0.8942(0.8899-0.8984)$ & $<0.0001$ \\
POAG & -0.17228 & $0.8175(0.7789-0.8584)$ & $<0.0001$ \\
Peptic ulcer drug & -0.27303 & $0.7131(0.6697-0.7593)$ & $<0.0001$ \\
Ischemic heart disease drug & -0.29949 & $0.8199(0.7701-0.8728)$ & $<0.0001$ \\
Depression drug & -0.4121 & $0.4873(0.4571-0.5195)$ & $<0.0001$ \\
Allergies & -0.57812 & $0.3590(0.3223-0.3997)$ & $<0.0001$ \\
Cancer drug & -2.23632 & $0.0862(0.0816-0.0911)$ & $<0.0001$ \\
\hline
\end{tabular}

Cl confidence interval, $O R$ odd ratio, $P O A G$ primary open angle glaucoma

comorbidity was diabetes in both, the GS (14.6\%) and non-GS cohort (13.6\%). Diabetes was followed by allergy, hypertension, hyperlipidemia and Ischemic heart disease. Allergy was remarkably higher in the GS cohort (Fig. 2a). Concomitant drugs use with frequency $>10 \%$ and significant difference in the two cohorts were hypertension, Ischemic heart disease, heart failure, stroke, depression, mental disorders, cancer and peptic ulcer. The use of cancer drug was remarkably higher in the GS cohort. (Fig. 2b). On the other hand, mitomycin C (MMC) originally was used as a systemic chemotherapeutic agent, and it has been used widely in ophthalmic practice, during and after surgery, for enhancing the success rate of glaucoma filtration surgery. Since concomitant drugs were limited to oral drugs and were excluded drugs used in the day of surgery, this study did not include MMC in the cancer drugs.

Among the GS cohort, the rate of comorbidities and the rate of concomitant use of prescribed systemic oral drugs in the CS and non-CS cohorts are shown in Fig. 3. The most common comorbidity was diabetes in both, the CS (17.7\%) and non-CS cohort

Table 4 Logistic regression analysis of factors associated with or without combined trabeculectomy and cataract surgery

\begin{tabular}{llll}
\hline Variable & Estimate & Adjusted OR (95\% CI) & $P$ Value \\
\hline Age $\leq 39$ & 2.62523 & $1.2066(6.2634-25.5751)$ & $<0.0001$ \\
Age 40-49 & 1.603633 & $47,396(3.4675-6.4783)$ & $<0.0001$ \\
Age 50-59 & 0.965275 & $2.6394(2.2454-3.1026)$ & $<0.0001$ \\
Cancer drug & 0.384652 & $1.4361(1.3062-1.5789)$ & $<0.0001$ \\
Hyperlipidemia drug & 0.194787 & $1.2737(1.0657-1.5222)$ & $<0.0001$ \\
POAG & -0.17353 & $0.8450(0.7761-0.9199)$ & $<0.0001$ \\
Age 70-79 & -0.28579 & $0.5642(0.5184-0.6140)$ & $<0.0001$ \\
\hline
\end{tabular}

$\mathrm{Cl}$ confidence interval, $\mathrm{OR}$ odd ratio, $P O A G$ primary open angle glaucoma
(19.3\%). Diabetes was followed by allergy and hypertension; however, the lack of significant differences was shown between the two cohorts (Fig. 3a). Concomitant drugs use with frequency $>10 \%$ and significant difference in the two cohorts were depression, mental disorders, and cancer (Fig. 3b).

The treatment patterns of prescribed topical glaucoma drugs (eye drops) at the index admission are summarized in Figs. 4 and 5. In the GS cohort, PG accounted for one third of total glaucoma eye drops. PG [2827 $(23.5 \%)]$ was followed by AA [1640 (13.3\%)], CAI [1086 (9.0\%)] and BB [713 (5.9\%)] by drug class; the most commonly used as first-line treatment was bimatoprost (8.9\%) followed by latanoprost (7.6\%), travoprost (3.1\%) and tafluprost $(3.0 \%)$ by generic name, including only original drugs not generic drugs (Fig. 4a). In the non-GS cohort, which has the similar treatment pattern to the GS cohort, PG [3923 (22.3\%)], AA [2317 (13.2\%)], CAI [1445 (8.2\%)] and BB [1212 (6.9\%)] were used by drug class, and the most commonly used as first-line treatment was bimatoprost (7.3\%), followed by latanoprost (7.2\%), tafluprost (3.8\%) and travoprost (3.1\%) by generic name (Fig. 4b). In the CS cohort, PG accounted for one third of total glaucoma eye drops. PG [673 (29.2\%)] was followed by AA [372 (16.2\%)], CAI [301 (13.1\%)] and BB [203 (8.8\%)] by drug class; the most commonly used as first-line treatment was latanoprost $(10.2 \%)$, followed by bimatoprost (9.3\%), travoprost (4.4\%) and tafluprost $(4.1 \%)$ by generic name (Fig. $5 \mathrm{a})$. In the non-CS cohort, which has the similar treatment pattern to the CS cohort, PG [2154 (30.7\%)], AA [1232 (17.6\%)], CAI [785 (11.2\%)] and ROCK [557 (7.9\%)] were used by drug class, and the most commonly used as first-line treatment was bimatoprost (12.2\%), followed by latanoprost (9.7\%), travoprost (3.9\%) and tafluprost (3.9\%) by generic name (Fig. 5b). 


\section{A}

Per 1000 population

160

140
120
100
80
60
40
20
0

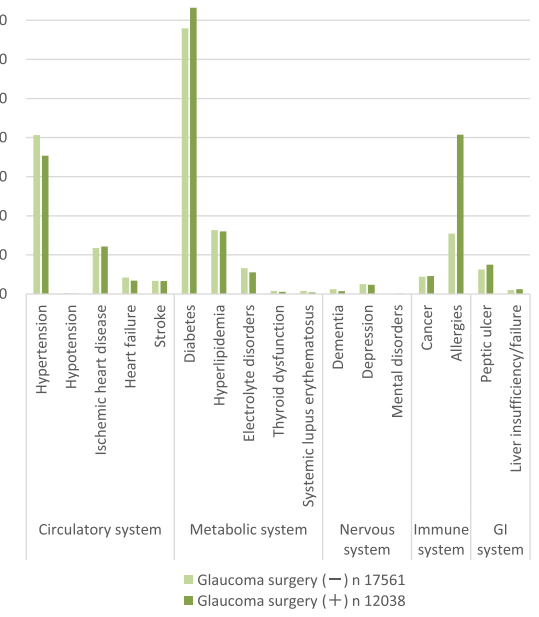

B

Per 1000 population

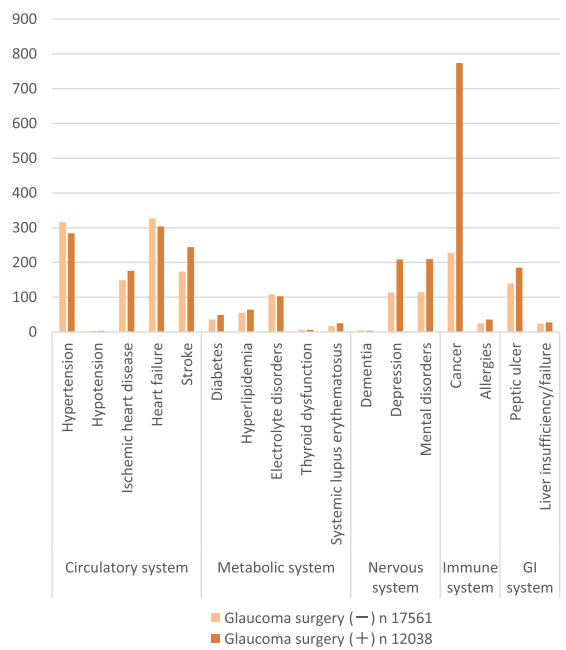

Fig. 2 a Prevalence rate of comorbidities, $\mathbf{b}$ rate of concomitant use of prescribed systemic oral drugs in the glaucoma surgery and nonglaucoma surgery cohorts

\section{Discussion}

To date, we found no other studies that identified the risk factors associates with TLE in patients with glaucoma in Japan or other countries. Our study did and revealed a significant influence of 11variables on TLE extracted from the claims database. Of these, 7 were significant more likely to increase TLS, and 4 were significant more likely to decrease TLE. The immune-related comorbidities and concomitant drugs use were the most likely to be TLE. Although there was no difference in the treatment pattern of prescribed glaucoma eye drops between the GS cohort and the non-GS cohort, the rate of the comorbidities and the rate of concomitant drugs use were similar trend to the above identified variables. Therefore, careful management of glaucoma patients with these variables may be important factor for reducing glaucoma surgery burden.

In Japan, TLO is also often preferred as glaucoma surgery, and TLE may target relatively severe glaucoma patients. Since this study used DPC data collected form

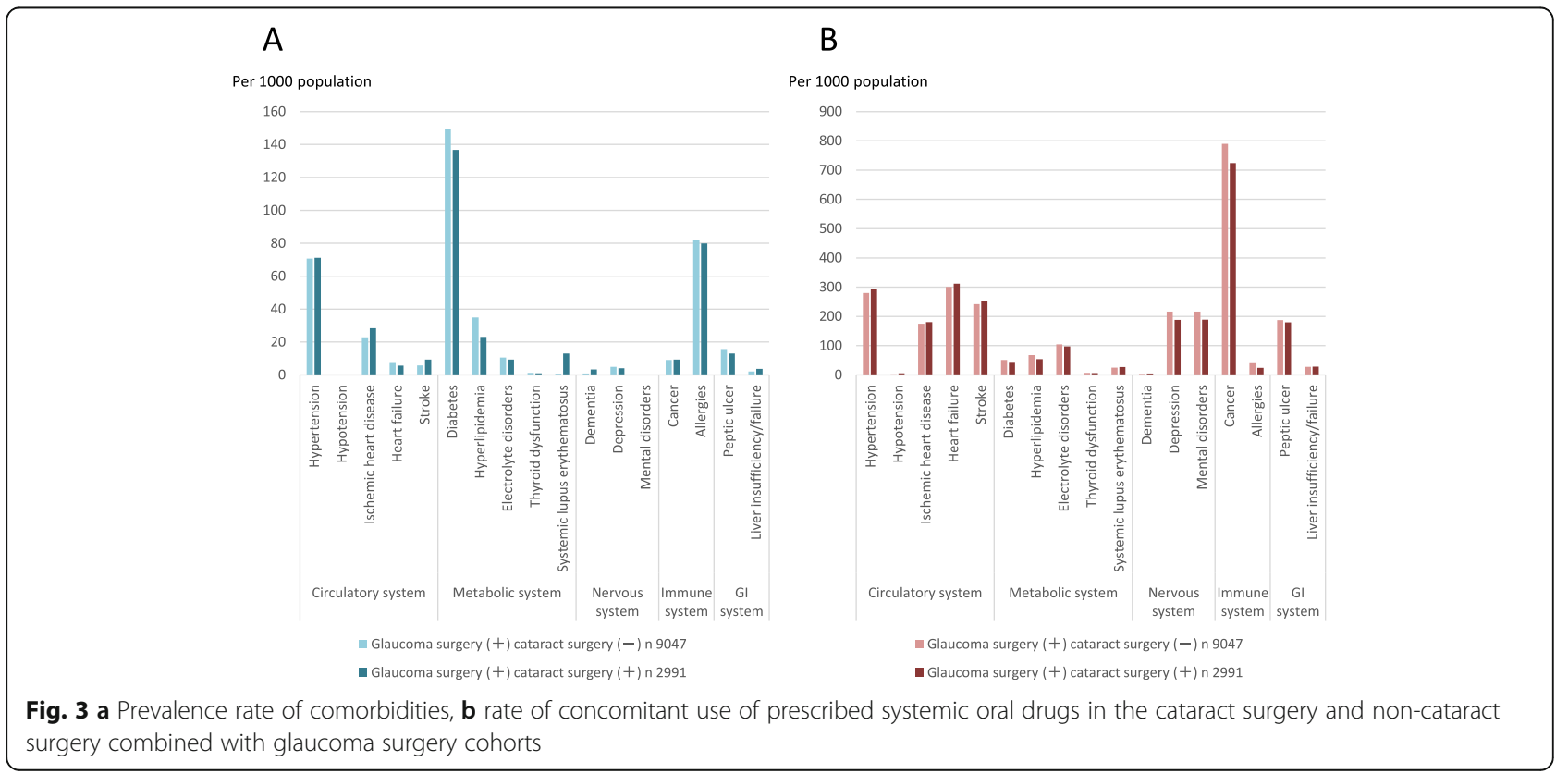



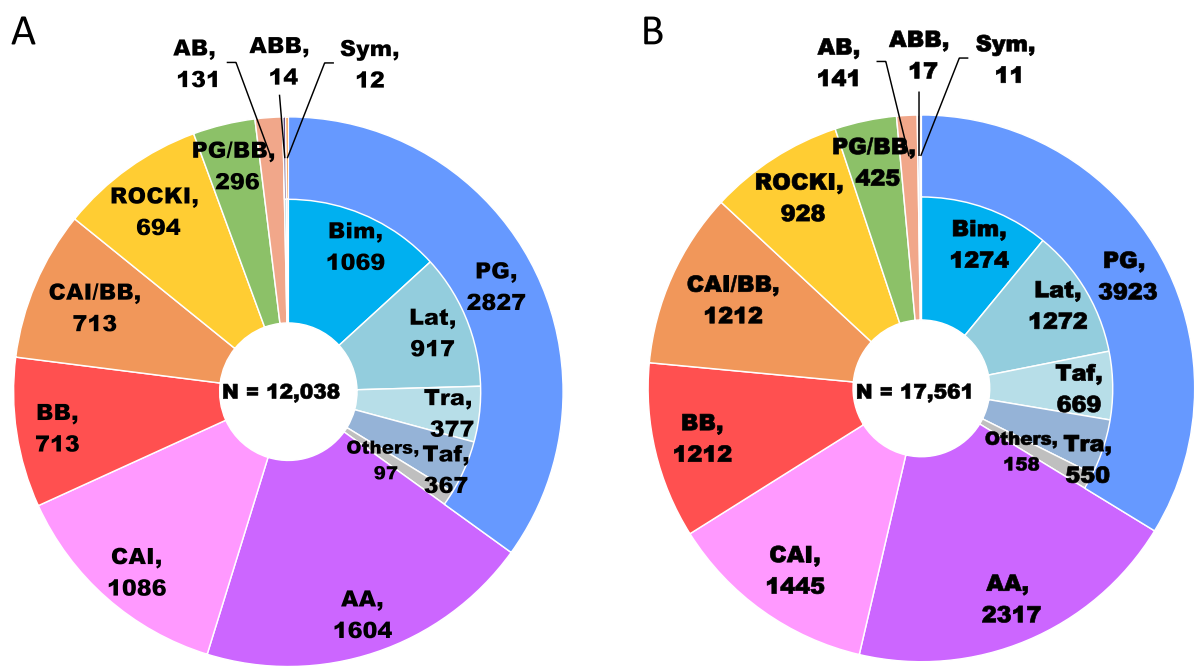

Fig. 4 Treatment patterns of prescribed topical glaucoma drugs (eye drops) at the index admission in the glaucoma surgery (a) and nonglaucoma surgery (b) cohorts. AA, a2-agonist; AB. a1-blocker; ABB, aß-blocker; BB, $\beta$-blocker; Bim, bimatoprost; CAl, carbonic anhydrase inhibitor; Lat, latanoprost; PG, prostaglandin analog; ROCKI, Rho-associated protein kinase inhibitor; Sym, sympathomimetics; Taf, tafluprost; Tra, travoprost

patients who were admitted to the nationwide acute care hospitals, we assumed that patients with relatively severe glaucoma were included and considered appropriate to select only TLE among GS. On the other hand, since TLO such as microhook TLO is often additionally used during CS, it is difficult to be an indicator for estimating exacerbation risk of glaucoma.

Among the 17 disease as the possible risk factors for glaucoma [23-26] (see Variable in the Method section), allergies were identified as the risk factors of comorbidities leading to TLE. Our finding might be explained by the previous studies: the toll-like receptor 4 (TLR4), a transmembrane receptor that mediates immune responses to exogenous, is associated with the risk of NTG [30]. The microglia are related to the immunocompetent cells of the central nervous system, and microglial activation has been reported in glaucoma [31], which might contribute to a higher prevalence of immune-related comorbidities such as allergies. Use of steroids by any route, however, can lead to increased IOP and can cause optic neuropathy resulting in steroidinduced glaucoma [32, 33]. Although our study included
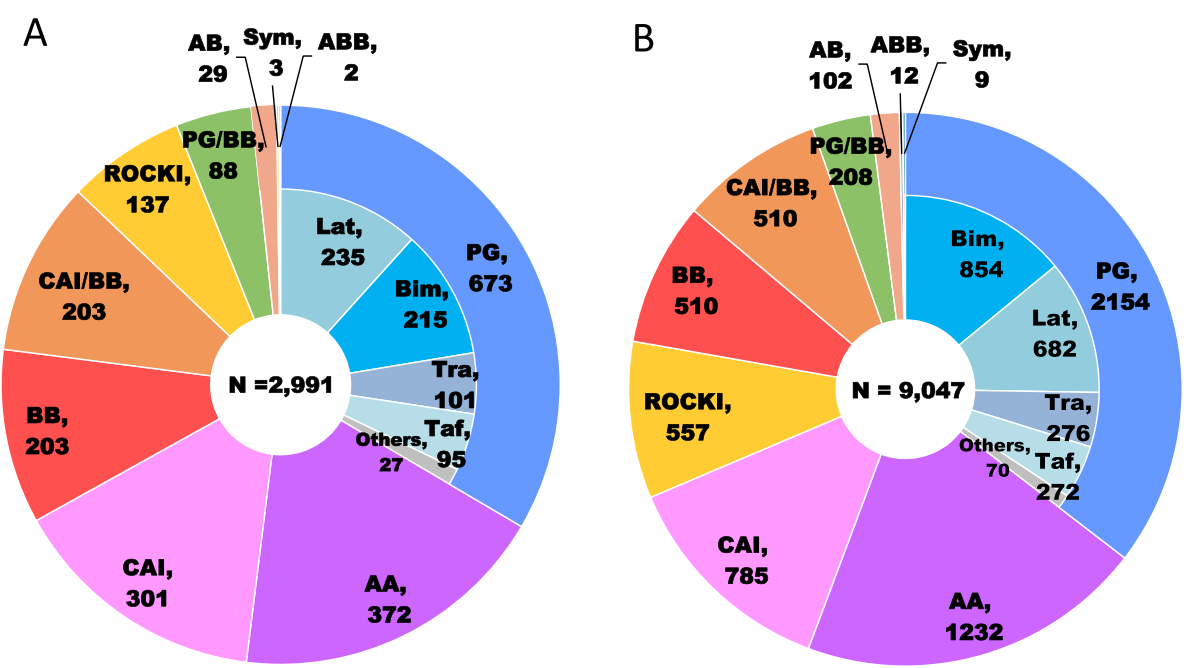

Fig. 5 Treatment patterns of prescribed topical glaucoma drugs (eye drops) at the index admission in the cataract surgery (a) and non-cataract surgery (b) combined with glaucoma surgery cohorts. AA, a2-agonist; AB. a1-blocker; ABB, aß-blocker; BB, $\beta$-blocker; Bim, bimatoprost; CAl, carbonic anhydrase inhibitor; Lat, latanoprost; PG, prostaglandin analog; ROCKI, Rho-associated protein kinase inhibitor; Sym, sympathomimetics; Taf, tafluprost; Tra, travoprost 
oral prednisolone use during the admissions (approximately $2.0 \%$ ), a history of corticosteroids use was excluded. Also, steroid-induced glaucoma (ICD-10: H406) was excluded so that corticosteroids as a potential confounding factor would be less influence between allergies and glaucoma.

Among systemic oral drugs for the 17 disease, cancer, depression, ischemic heart disease or peptic ulcer were identified as the risk factors of concomitant drugs leading to GS. According to the previous studies, the downregulation of cell cycle progression by checkpoint inhibitors has recently been targeting for cancer therapy $[34,35]$, can cause cell death beyond cancer cells, and therefore may induce neurodegenerative disorders such as glaucoma [36]. However, one of the risk factors for glaucoma is increased oxidative stress, and drugs targeting oxidative stress in cancer could reduce the oxidative stress-induced apoptosis of retinal ganglion cells in glaucoma $[37,38]$. Therefore, we have not been able to identify reports from previous studies of whether cancer drugs is associated with TLE. According to Table 2, only 110 patients in the GS cohort (0.9\%) and 155 patients in the non-GS cohort (0.9\%) had cancer. However, 9313 patients in the GS cohort (77.4\%) and 3997 patients in the non-GS cohort (22.8\%) used medications for cancer. From these, the GS cohort used more medication for cancer than the non-GS cohort and would be considered more sever stage of glaucoma. Since pharmacy codes that used medications for cancer include 421 (alkylating agents), 422 (antimetabolites), 423 (antibiotics), and 424 (plant extract preparations), cancer treatments may have been used not only for cancer patients but also noncancer patients.

Other studies report that depression is strongly linked with glaucoma [39] and results in elevated oxidative stress [40]. Likewise, ischemic heart disease is linked with glaucoma probably affect vasculature dysfunction [41]. Furthermore, many factors contribute to peptic ulcer including glaucoma [42] is another likely reason that may contribute to the positive association. In contrast, hypertension and its drug were identified as the risk factors leading to non-GS. Our finding might to be in line with the previous studies that hypertension improve ocular blood flow [43], however, hypertension oral administration is a risk of glaucoma progression [44]. Stratified analysis based in CS showed that none of the 17 disease were identified as the risk factors leading to CS combined with TLE.

In addition, patients with POAG was also identified as the significant more likely to be TLE. The rate for glaucoma type in subjects 40 years of age and older was estimated at $5.0 \%$ for all glaucoma, $0.3 \%$ for POAG, and $3.6 \%$ for NTG [5]. In contrast, this study showed that the rates of POAG, OAG and NTG at the index admission were $36.6,35.0$ and $6.2 \%$, respectively, in the GS cohort, and 32.0, 36.0 and $7.6 \%$, respectively, in the non-GS cohort. The ratios of NTG seem to be fewer than expected. One possible explanation could be that the rate of progress of glaucomatous optic neuropathy among patients with NTG is generally slower than that among those with other types of glaucoma, therefore, patients with NTG have less need to go to DPC hospitals.

Furthermore, this study identified longer LOS as the factors associate with TLE. Japanese hospitals generally provide rehabilitation and nursing care in addition to acute medical care, which may contribute to the longer LOS.

Patients in both cohorts, the high rate of comorbidities and concomitant drugs use were similar trend to the above identified variables. In the GS cohort, allergy and cancer drugs were most significantly higher than the non-GS cohort. Diabetes was the most common comorbidities in both, the GS (14.6\%) and non-GS cohort (13.6\%), and significantly higher in the GS cohorts, but not identified as the risk factors leadings to GS. On the other hand, the rate of the diabetes drug use was low in both, the GS (4.9\%) and non-GS cohort (3.6\%). Since the diabetes drug has been reported as both a risk factor and a protective factor for glaucoma [23-26], this study show that few patients with glaucoma who had diabetes may be treated with the diabetes drug.

The treatment patterns of prescribed glaucoma eye drops at the index admission was similar between the two cohorts; PG was most commonly prescribed, AA and CAI were second and third, respectively. Our result is in line with the data from a published report indicating that the most commonly used first-line monotherapy was a PG [45-48], while CAI/BB was the most commonly used fixed combination as first- and second-line treatment $[49,50]$. Although BB is also recommended as a first-line monotherapy in the guideline for glaucoma, the prescription rate of $\mathrm{BB}$ was low in this study. This is probably because the patients had comorbidities of asthmas, chronic obstructive pulmonary disease or heart failure may not prescribed $\mathrm{BB}$ according to the respective drug information. Or elderly patients may have difficult using BB. On the other hand, BB was considered to be common in the GS cohorts because of bradycardia, but not so in our results. The ROCKI as well as the EP2 receptor agonist become available in Japan recently and has been reported to show an additional IOP-lowering in combination with other glaucoma ophthalmic solutions [51-54]. Thus, the prescription trend for glaucoma eye drops may change in the future.

This study had several limitations. First, we included only DPC hospitals with glaucoma beds, so the results may not be generalizable; however, DPC database 
contains detailed medical data on numerous patients residing throughout the Japan in a broad array of geographic regions. Moreover, the variables included in the final predictive model are available in other Japanese administrative claims databases. Second, limitations common to studies using administrative claims data apply to this study [55-58]. These limitations include lack of certain information in the database and errors or omissions in claims coding. Third, claims data lack clinical information (such as IOP, visual field, etc.) to access disease severity. Therefore, it was not possible to evaluate whether the severity level of documented comorbid conditions was comparable between our study cohorts and whether different stages of glaucoma were associated with specific comorbidity profiles. Fourth, our data did not exclude laser trabeculoplasty (LT). Although, LT reported to be an alternative to topical glaucoma drug treatment and the same IOP-lowering effect as eye drops as monotherapy [59], it may have influenced the results of our analysis. Fifth, unmeasured confounders may limit the findings. Finally, the present analyses were built according to the assumption that all the claimed drugs were used by the patients. To address these limitations, we need to conduct further studies using the real-world data combined with clinical data.

\section{Conclusions}

The results of this study show that the risk factors leading to TLE of glaucoma patient can be predicted with 7 commonly available demographic and administrative claim-based variables including: having comorbidities related to allergies; taking concomitant drugs related to cancer, depression, ischemic heart disease and peptic ulcer; being diagnosed with POAG, and longer LOS. Furthermore, the rate of comorbidities and the rate of concomitant drug use were similar tendency as the above identified variables. Therefore, before starting glaucoma treatment, special focus on Japanese patients with glaucoma, especially POAG, who have allergyrelated comorbidities, or take the immune, nervous, circulatory or gastrointestinal system-related concomitant drugs, through medical interview by ophthalmologists seems to be desirable.

\footnotetext{
Abbreviations

AA: a2 agonist; $A B$ : a1 blocker; $A B B$ : $a \beta$ blocker; BB: $\beta$ blocker; BMl: Body mass index; CAl: Carbonic anhydrase inhibitor; $\mathrm{CCl}$ : Charlson comorbidity index; Cls: Confidence intervals; CS: Cataract surgery; DPC: Diagnosis procedure combination; GS: Glaucoma surgery; ICD: International statistical classification of disease; IOP: Intraocular pressure; LOS: Length of stay in hospital; LT: Laser trabeculoplasty; NTG: Normal tension glaucoma; OAG: Open angle glaucoma; OR: Odds ratio; PG: Prostaglandin analog; POAG: Primary open angle glaucoma; ROCKI: Rho-associated coiled kinase inhibitor; SD: Standard deviation; TLO: Trabeculotomy; TLE: Trabeculectomy; VIF: Variance inflation factor
}

\section{Supplementary Information}

The online version contains supplementary material available at https://doi. org/10.1186/s12886-021-01897-4.

Additional file 1: Supplemental Table 1. Baseline demographic and clinical characteristics of the study subgroups.

\section{Acknowledgments}

This study was supported by a Grant-in-Aid for Research on Policy Planning and Evaluation from the Ministry of Health, Labour and Welfare, Japan.

\section{Authors' contributions}

CS, ST, KF and TN were involved in the conceptual development and study design. KF and KF developed the database and CS, KT and KF performed the statistical analysis. All authors participated in the interpretation of data and in drafting and/or revising the manuscript critically for developing important intellectual content. All authors read and approved the final manuscript.

\section{Funding}

Not applicable.

\section{Availability of data and materials}

The data that support the findings of this study are available from the DPC Study Group, a government-funded academic group, but restrictions apply to the availability of these data, which were used under license for the current study. Therefore, the data are not publicly available. The data are however available from the authors upon reasonable request and with the permission of the DPC Study Group.

\section{Declarations}

Ethics approval and consent to participate

This study was conducted in accordance with the Declaration of Helsinki and was approved by the research ethics committee of the Tohoku University Graduate School of Medicine, Japan (No. 2019-1-897). This study acquired secondary data from a medical claims database, written informed consent from patients was not required.

Consent for publication

Not applicable.

\section{Competing interests}

The authors declare that they have no competing interests.

\section{Author details}

'Department of Ophthalmology, Tohoku University Graduate School of Medicine, 1-1 Seiryo-machi, Aoba-ku, Sendai, Miyagi 980-8574, Japan. ${ }^{2}$ Department of Health Administration and Policy, Tohoku University Graduate School of Medicine, 1-1 Seiryo-machi, Aoba-ku, Sendai, Miyagi 980-8574, Japan. ${ }^{3}$ Department of Health Policy and Informatics, Tokyo Medical and Dental University Graduate School of Medicine, Tokyo, Japan.

Received: 8 November 2020 Accepted: 9 March 2021

Published online: 29 March 2021

References

1. Quigley HA, Broman AT. The number of people with glaucoma worldwide in 2010 and 2020. Br J Ophthalmol. 2006;90(3):262-7. https://doi.org/10.113 6/bjo.2005.081224.

2. Parihar JKS. Glaucoma: the 'black hole' of irreversible blindness. Med J Armed Forces India. 2016;72(1):3-4. https://doi.org/10.1016/j.mjafi.2015.12.001.

3. Nordstrom BL, Friedman DS, Mozaffari E, Quigley HA, Walker AM. Persistence and adherence with topical glaucoma therapy. Am J Ophthalmol. 2005;140(4):598-606. https://doi.org/10.1016/j.ajo.2005.04.051.

4. Garway-Heath DF, Crabb DP, Bunce C, Lascaratos G, Amalfitano F, Anand N, Azuara-Blanco A, Bourne RR, Broadway DC, Cunliffe IA, Diamond JP, Fraser SG, Ho TA, Martin KR, McNaught Al, Negi A, Patel K, Russell RA, Shah A, Spry PG, Suzuki K, White ET, Wormald RP, Xing W, Zeyen TG. Latanoprost for open-angle glaucoma (UKGTS): a randomised, multicentre, placebo- 
controlled trial. Lancet. 2015;385(9975):1295-304. https://doi.org/10.1016/ S0140-6736(14)62111-5.

5. The Japan glaucoma society guidelines for glaucoma (4th edition). Nippon Ganka Gakkai Zasshi 2018; 122:5-53. http://www.nichigan.or.jp/member/ guideline/glaucoma4.pdf. Last accessed on 8 Nov 2020. (in Japanese).

6. Morizane Y, Morimoto N, Fujiwara A, Kawasaki R, Yamashita H, Ogura Y, Shiraga F. Incidence and cause of visual impairment in Japan: the first nation-wide complete enumeration survey of newly certified visually impaired individuals. Jpn J Ophthalmol. 2019;63(1):26-33. https://doi.org/1 0.1007/s10384-018-0623-4.

7. Iwase A, Suzuki Y, Araie M, Yamamoto T, Abe H, Shirato S, Kuwayama $Y$, Mishima HK, Shimizu H, Tomita G, Inoue Y, Kitazawa Y, Tajimi Study Group, Japan Glaucoma Society. The prevalence of primary open-angle glaucoma in Japanese: the Tajimi study. Ophthalmology. 2004;111(9):1641-8. https:// doi.org/10.1016/j.ophtha.2004.03.029.

8. Coleman AL, Miglior S. Risk factors for glaucoma onset and progression. Surv Ophthalmol. 2008;53:3-10.

9. Tham YC, Li X, Wong TY, Quigley HA, Aung T, Cheng CY. Global prevalence of glaucoma and projections of glaucoma burden through 2040: a systematic review and meta-analysis. Ophthalmology. 2014;121(11):2081-90. https://doi.org/10.1016/j.ophtha.2014.05.013.

10. Richter GM, Coleman AL. Minimally invasive glaucoma surgery: current status and future prospects. Clin Ophthalmol. 2016;10:189-206. https://doi. org/10.2147/OPTH.S80490.

11. Bovee CE, Pasquale LR. Evolving surgical interventions in the treatment of glaucoma. Semin Ophthalmol. 2017;32(1):91-5. https://doi.org/10.1080/0882 0538.2016 .1228393$.

12. Chen DZ, Sng CCA. Safety and efficacy of microinvasive glaucoma surgery. J Ophthalmol. 2017; Article ID 3182935:13.

13. Masuda S. Casemix as a tool for transparency of medical service. Jpn J Secur Policy. 2007;6:43-53.

14. Fushimi K, Hashimoto H, Imanaka Y, Kuwabara K, Horiguchi H, Ishikawa KB, Matsuda S. Functional mapping of hospitals by diagnosis-dominant casemix analysis. BMC Health Serv Res. 2007;7(1):50. https://doi.org/10.1186/14 72-6963-7-50.

15. Matsuda S. Case mix as a tool for transparency of medical services. Jpn J Secur Policy. 2017:6:11.

16. Yasuda S, Nakao K, Nishimura K, Miyamoto Y, Sumita Y, Shishido T, Anzai T, Tsutsui H, Ito H, Komuro I, Saito Y, Ogawa H, on the behalf of JROAD Investigators. The current status of cardiovascular medicine in Japan: analysis of a large number of health records from a nationwide claim-based database, JROAD-DPC. Circ J. 2016;80(11):2327-35. https://doi.org/10.1253/ circj.CJ-16-0196

17. Yasunaga $\mathrm{H}$, Ide $\mathrm{H}$, Imamura $\mathrm{T}$, Ohe $\mathrm{K}$. Impact of the Japanese diagnosis procedure combination-based payment system on cardiovascular medicinerelated costs. Int Heart J. 2005;46(5):855-66. https://doi.org/10.1536/ihj.46.855.

18. Nakamura K. Diagnosis procedure combination database would develop nationwide clinical research in Japan. Circ J. 2016;80(11):2289-90. https://doi. org/10.1253/circj.CJ-16-0973.

19. Tarasawa K, Fujimori K, Fushimi K. Recombinant human soluble thrombomodulin contributes to a reduction in-hospital mortality of acute cholangitis with disseminated intravascular coagulation: a propensity score analyses of a Japanese nationwide database. Tohoku J Exp Med. 2020; 252(1):53-61. https://doi.org/10.1620/tjem.252.53.

20. lihara K, Nishimura K, Kada A, Nakagawara J, Toyoda K, Ogasawara K, Ono J, Shiokawa Y, Aruga T, Miyachi S, Nagata I, Matsuda S, Ishikawa KB, Suzuki A, Mori H, Nakamura F, J-ASPECT Study Collaborators. The impact of comprehensive stroke care capacity on the hospital volume of stroke interventions: a nationwide study in Japan: J-ASPECT study. J Stroke Cerebrovasc Dis. 2014;23(5):1001-18. https://doi.org/10.1016/j. jstrokecerebrovasdis.2013.08.016.

21. Charlson ME, Pompei P, Ales KL, Mackenzie CR. A new method of classifying prognostic comorbidity in longitudinal studies: development and validation. J Chronic Dis. 1987;40(5):373-83. https://doi.org/10.1016/0021-9681(87)90171-8.

22. Sundararajan V, Quan H, Halfon P, Fushimi K, Luthi JC, Burnand B, Ghali WA, International Methodology Consortium for Coded Health Information (IMEC CHI). Cross-national comparative performance of three versions of the ICD10 Charlson index. Med Care. 2007:45(12):1210-5. https://doi.org/10.1097/ MLR.0b013e3181484347.

23. Ho H, Shi Y, Chua J, Tham YC, Lim SH, Aung T, Wong TY, Cheng CY. Association of systemic medication use with intraocular pressure in a multiethnic Asian population: the Singapore epidemiology of eye diseases study. JAMA Ophthalmol. 2017;135(3):196-202. https://doi.org/10.1001/jama ophthalmol.2016.5318.

24. Chen VC, Ng M, Chiu W, et al. Effects of selective serotonin reuptake inhibitors on glaucoma: a nationwide population-based study. PLoS One. 2017;12(3):e0173005. https://doi.org/10.1371/journal.pone.0173005.

25. Chen HY, Hsu SY, Chang YC, et al. Association between statin use and open-angle glaucoma in hyperlipidemia patients: a Taiwanese populationbased case-control study. Medicine (Baltimore). 2015;94:e2018.

26. Shiga Y, Akiyama M, Nishiguchi KM, Sato K, Shimozawa N, Takahashi A, Momozawa Y, Hirata M, Matsuda K, Yamaji T, Iwasaki M, Tsugane S, Oze I, Mikami H, Naito M, Wakai K, Yoshikawa M, Miyake M, Yamashiro K, Japan Glaucoma Society Omics Group (JGS-OG), Kashiwagi K, Iwata T, Mabuchi F, Takamoto M, Ozaki M, Kawase K, Aihara M, Araie M, Yamamoto T, Kiuchi Y, Nakamura M, Ikeda Y, Sonoda KH, Ishibashi T, Nitta K, Iwase A, Shirato S, Oka Y, Satoh M, Sasaki M, Fuse N, Suzuki Y, Cheng CY, Khor CC, Baskaran M, Perera S, Aung T, Vithana EN, Cooke Bailey JN, Kang JH, Pasquale LR, Haines $J L$, NEIGHBORHOOD Consortium, Wiggs $J L$, Burdon KP, Gharahkhani P, Hewitt AW, Mackey DA, MacGregor S, Craig JE, Allingham RR, Hauser M, Ashaye A, Budenz DL, Akafo S, Williams SEl, Kamatani Y, Nakazawa T, Kubo M. Genome-wide association study identifies seven novel susceptibility loci for primary open-angle glaucoma. Hum Mol Genet. 2018;27(8):1486-96. https://doi.org/10.1093/hmg/ddy053.

27. Gueugnon M, Stapley PJ, Gouteron A, Lecland C, Morisset C, Casillas JM, Ornetti P, Laroche D. Age-related adaptations of lower limb intersegmental coordination during walking. Front Bioeng Biotechnol. 2019;7:173. https:// doi.org/10.3389/fbioe.2019.00173.

28. Aizawa H, Imai S, Fushimi K. Factors associated with 30-day readmission of patients with heart failure from a Japanese administrative database. BMC Cardiovasc Disord. 2015;15(1):134. https://doi.org/10.1186/s12872-015-0127-9.

29. Hayashi T, Boyko EJ, Leonetti DL, McNeely MJ, Newell-Morris L, Kahn SE, Fujimoto WY. Visceral adiposity is an independent predictor of incident hypertension in Japanese Americans. Ann Intern Med. 2004;140(12):9921000. https://doi.org/10.7326/0003-4819-140-12-200406150-00008.

30. Shibuya E, Meguro A, Ota M, Kashiwagi K, Mabuchi F, lijima H, Kawase K, Yamamoto T, Nakamura M, Negi A, Sagara T, Nishida T, Inatani M, Tanihara H, Aihara M, Araie M, Fukuchi T, Abe H, Higashide T, Sugiyama K, Kanamoto T, Kiuchi Y, Iwase A, Ohno S, Inoko H, Mizuki N. Association of toll-like receptor 4 gene polymorphisms with normal tension glaucoma. Invest Ophthalmol Vis Sci. 2008;49(10):4453-7. https://doi.org/10.1167/iovs.07-1575.

31. Ramirez Al, de Hoz R, Salobrar-Garcia E, et al. The role of microglia in retinal neurodegeneration: alzheimer's disease, parkinson, and glaucoma. Front Aging Neurosci. 2017;9:1-21.

32. McLean JM. Use of ACTH and cortisone. Trans Am Ophthalmol Soc. 1950;48: 293-6.

33. Francois J. Cortisone et tension oculaire. Ann D'Oculist. 1954;187:805.

34. Visconti R, Monica RD, Grieco D. Cell cycle checkpoint in cancer: a therapeutically targetable double-edged sword. J Exp Clin Cancer Res. 2016; 35(1):153. https://doi.org/10.1186/s13046-016-0433-9.

35. Benada J, Macurek L. Targeting the checkpoint to kill cancer cells. Biomolecules. 2015;5(3):1912-37. https://doi.org/10.3390/biom5031912.

36. Golubnitschaja O. Cell cycle checkpoints: the role and evaluation for early diagnosis of senescence, cardiovascular, cancer, and neurodegenerative diseases. Amino Acids. 2007;32(3):359-3571. https://doi.org/10.1007/s00726006-0473-0.

37. Kimura A, Namekata N, Guo X, et al. Targeting oxidative stress for treatment of glaucoma and optic neuritis. Oxidative Med Cell Longev. 2017;2817252.

38. Wang C, Ren YL, Zhai J, Zhou XY, Wu J. Down-regulated LAMA4 inhibits oxidative stress-induced apoptosis of retinal ganglion cells through the MAPK signaling pathway in rats with glaucoma. Cell Cycle. 2019;18(9):93248. https://doi.org/10.1080/15384101.2019.1593645.

39. Chen YY, Lai YJ, Wang JP, Shen YC, Wang CY, Chen HH, Hu HY, Chou P. The association between glaucoma and risk of depression: a nationwide population-based cohort study. BMC Ophthalmol. 2018;18(1):146. https://doi. org/10.1186/s12886-018-0811-5.

40. Gislaine Z, Réus AS, Carlessi RH, et al. Relationship of oxidative stress as a link between diabetes mellitus and major depressive disorder. Oxidative Med Cell Longev. 2019:e8637970.

41. Chen YY, Hu HY, Chu D, Chen HH, Chang CK, Chou P. Patients with primary open-angle glaucoma may develop ischemic heart disease more often than 
those without glaucoma: an 11-year population-based cohort study. PLoS One. 2016;11(9):e0163210. https://doi.org/10.1371/journal.pone.0163210.

42. Knox DL. Glaucomatocyclitic crises and systemic disease: peptic ulcer, other gastrointestinal disorders, allergy and stress. Trans Am Ophthalmol Soc. 1988:86:473-95.

43. Caprioli J, Coleman AL. Blood flow in glaucoma discussion; blood pressure, perfusion pressure, and glaucoma. Am J Ophthalmol. 2010;149(5):704-12. https://doi.org/10.1016/j.ajo.2010.01.018.

44. Krupin T, Liebmann JM, Greenfield DS, Rosenberg LF, Ritch R, Yang JW, Low-Pressure Glaucoma Study Group. Low-Pressure Glaucoma Study Group. The Low-pressure Glaucoma Treatment Study (LoGTS) study design and baseline characteristics of enrolled patients. Ophthalmology. 2005;112(3): 376-85. https://doi.org/10.1016/j.ophtha.2004.10.034

45. Choudhari NS, Pathak-ray V, Kaushik S, Vyas P, George R. Understanding practice patterns of glaucoma sub- specialists in India. Int J Opthalmol. 2017;10:1580-5.

46. Coleman AL, Lum FC, Su Z, Gliklich RE. Practice patterns and treatment changes for open-angle glaucoma: the RiGOR study. J Comp Eff Res. 2016; 5(1):79-85. https://doi.org/10.2217/cer.15.57.

47. Marquis RE, Whitson JT. Management of glaucoma: focus on pharmacological therapy. Drugs Aging. 2005;22(1):1-21. https://doi.org/10.21 65/00002512-200522010-00001

48. Whitson JT. Glaucoma: a review of adjunctive therapy and new management strategies. Expert Opin Pharmacother. 2007;8:39-40.

49. Goldshtein I, Shalev V, Zigman N. The Maccabi glaucoma study: treatment patterns and persistence with glaucoma therapy in a large Israeli health maintenance organization. J Glaucoma. 2016;25:386-3891.

50. Kim CY, Park KH, Ahn J, Ahn MD, Cha SC, Kim HS, Kim JM, Kim MJ, Kim TW, Kim YY, Lee JW, Park SW, Sohn YH, Sung KR, Yoo C, Cha J, Kim YJ. Treatment patterns and medication adherence of patients with glaucoma in South Korea. Br J Ophthalmol. 2017;101(6):801-7. https://doi.org/10.1136/ bjophthalmol-2016-308505.

51. Tanihara H, Inoue T, Yamamoto T, Kuwayama $Y$, Abe H, Suganami H, Araie M, K-115 Clinical Study Group. Additive intraocular pressure-lowering effects of the rho kinase inhibitor ripasudil (K-115) combined with timolol or latanoprost: a report of 2 randomized clinical trials. JAMA Ophthalmol. 2015; 133(7):755-61. https://doi.org/10.1001/jamaophthalmol.2015.0525.

52. Yamamoto K, Maruyama K, Himori N, Omodaka K, Yokoyama Y, Shiga Y, Morin R, Nakazawa T. The novel rho kinase (ROCK) inhibitor K-115: a new candidate drug for neuroprotective treatment in glaucoma. Investig Ophthalmol. 2014;55(11):7126-36. https://doi.org/10.1167/iovs.13-13842.

53. Imamura R, Tanaka M, Okanari E, Kirihara T, Odani-Kawabata N, Shams N, Yoneda K. Identification of a selective, non-prostanoid EP2 receptor agonist for the treatment of glaucoma: omidenepag and its prodrug omidenepag isopropyl. J Med Chem. 2018;61(15):6869-91. https://doi.org/10.1021/acs. imedchem.8b00808

54. Aihara M, Lu F, Kawata H, Iwata A, Liu K, Odani-Kawabata N, Shams NK. Phase 2, randomized, dose-finding studies of omidenepag isopropyl, a selective EP2 agonist, in patients with primary open-angle glaucoma or ocular hypertension. J Glaucoma. 2019;28(5):375-85. https://doi.org/10.1097/ IJG.0000000000001221.

55. Grimes D. Epidemiologic research using administrative databases: garbage in, garbage out. Obstet Gynecol. 2010;116(5):1018-9. https://doi.org/10.1 097/AOG.0b013e3181f98300.

56. Hennessy S. Use of health care databases in pharmacoepidemiology. Basic Clin Pharmacol Toxicol. 2006;98(3):311-3. https://doi.org/10.1111/j.17427843.2006.pto 368.x

57. Hersh WR, Weiner MG, Embi PJ, et al. Caveats for the use of operational electronic health record data in comparative effectiveness research. Med Care. 2013;51:30-7.

58. Schneeweiss $\mathrm{S}$, Avorn J. A review of uses of health care utilizationdatabases for epidemiologic research on therapeutics. J Clin Epidemiol. 2005;58(4): 323-37. https://doi.org/10.1016/j.jclinepi.2004.10.012.

59. Gazzard G, Konstantakopoulou E, Garway-Heath D, et al. LiGHT Trial Study Group. Selective laser trabeculoplasty versus eye drops for first-line treatment of ocular hypertension and glaucoma (LiGHT): a multicentre randomised controlled trial. Lancet. 2019;393:1505-16.

\section{Publisher's Note}

Springer Nature remains neutral with regard to jurisdictional claims in published maps and institutional affiliations.

Ready to submit your research? Choose BMC and benefit from:

- fast, convenient online submission

- thorough peer review by experienced researchers in your field

- rapid publication on acceptance

- support for research data, including large and complex data types

- gold Open Access which fosters wider collaboration and increased citations

- maximum visibility for your research: over $100 \mathrm{M}$ website views per year

At BMC, research is always in progress.

Learn more biomedcentral.com/submissions 